\title{
Dynein Light Chain LC8 Regulates Syntaphilin-Mediated Mitochondrial Docking in Axons
}

\author{
Yan-Min Chen, Claudia Gerwin, and Zu-Hang Sheng \\ Synaptic Function Section, The Porter Neuroscience Research Center, National Institute of Neurological Disorders and Stroke-National Institutes of Health, \\ Bethesda, Maryland 20892-3706
}

\begin{abstract}
Mitochondria in the cell bodies of neurons are transported down neuronal processes in response to changes in local energy and metabolic states. Because of their extreme polarity, neurons require specialized mechanisms to regulate mitochondrial transport and retention in axons. Our previous studies using syntaphilin $(s n p h)$ knock-out mice provided evidence that SNPH targets to axonal mitochondria and controls their mobility through its static interaction with microtubules (MTs). However, the mechanisms regulating SNPH-mediated mitochondrial docking remain elusive. Here, we report an unexpected role for dynein light chain LC8. Using proteomic biochemical and cell biological assays combined with time-lapse imaging in live snph wild-type and mutant neurons, we reveal that LC8 regulates axonal mitochondrial mobility by binding to SNPH, thus enhancing the SNPH-MT docking interaction. Using mutagenesis assays, we mapped a seven-residue LC8-binding motif. Through this specific interaction, SNPH recruits LC8 to axonal mitochondria; such colocalization is abolished when neurons express SNPH mutants lacking the LC8-binding motif. Transient LC8 expression reduces mitochondrial mobility in snph $(+/+)$ but not $(-I-)$ neurons, suggesting that the observed effect of LC8 depends on the SNPH-mediated docking mechanism. In contrast, deleting the LC8-binding motif impairs the ability of SNPH to immobilize axonal mitochondria. Furthermore, circular dichroism spectrum analysis shows that LC8 stabilizes an $\alpha$-helical coiled-coil within the MT-binding domain of SNPH against thermal unfolding. Thus, our study provides new mechanistic insights into controlling mitochondrial mobility through a dynamic interaction between the mitochondrial docking receptor and axonal cytoskeleton.
\end{abstract}

\section{Introduction}

Proper transport and distribution of mitochondria within axons are critical for neuronal function. In addition to the aerobic production of ATP, mitochondria regulate $\mathrm{Ca}^{2+}$ concentrations (Werth and Thayer, 1994) and have been implicated in certain forms of short-term synaptic plasticity by buffering $\mathrm{Ca}^{2+}$ at synapses (Tang and Zucker, 1997; Billups and Forsythe, 2002; Levy et al., 2003; Yang et al., 2003; Kang et al., 2008). Because of their extreme polarity, neurons require specialized mechanisms to regulate mitochondrial transport and retention in the vicinity of active growth cones and branches in developing neurons, nodes of Ranvier, myelination boundaries, and synaptic terminals of

\footnotetext{
Received March 27, 2009; revised June 3, 2009; accepted June 20, 2009.

This work was supported by the National Institute of Neurological Disorders and Stroke (NINDS) Intramural Competitive Fellowship Award (Y.-M.C.) and the Intramural Research Program of NINDS, National Institutes of Health (NIH) (Z.-H.S.). Y.-M.C. conducted the majority of the cell biology and biochemical studies and manuscript writing; C.G. performed some of the biochemical analysis. Z.-H.S. is a senior author who was responsible for the project design and revised this manuscript. The animal care and use in this study were in accordance with the NIH guidelines and was approved by the NIH-NINDS-National Institute on Deafness and Other Communication Disorders (NIDCD) Animal Care and Use Committee. We thank the following people for their help: J.-S. Kang and Q. Cai for their suggestions on live-cell imaging; $\mathrm{Q}$. Cai for drawing the schematic model; H. Jaffe (Protein Sequencing Facility of NINDS, NIH) for mass spectrometry, D. Krepkiy (the Swartz laboratory) for CD spectroscopy, and D. GlazerSchoenberg (NINDS) for editing.

The authors declare no competing financial interests.

Correspondence should be addressed to Zu-Hang Sheng, Synaptic Function Section, the Porter Neuroscience Research Center, National Institute of Neurological Disorders and Stroke-National Institutes of Health, Building 35, Room 2B215, 35 Convent Drive, Bethesda, MD 20892-3706. E-mail: shengz@ninds.nih.gov.

D01:10.1523/JNEUROSCI.1472-09.2009

Copyright $\odot 2009$ Society for Neuroscience $\quad$ 0270-6474/09/299429-10\$15.00/0
}

mature neurons, where energy production and calcium homeostasis capacity are in high demand (Hollenbeck and Saxton, 2005).

In neurons, long-distance fast axonal transport of mitochondria depends on microtubule (MT)-based motor proteins (Hollenbeck, 1996). Whereas kinesin motors are responsible for anterograde transport of axonal mitochondria, cytoplasmic dynein motors are the driving force behind retrograde movement (Tanaka et al., 1998; Martin et al., 1999; Ligon and Steward, 2000; Stowers et al., 2002; Górska-Andrzejak et al., 2003; Cai et al., 2005; Guo et al., 2005; Glater et al., 2006; Pilling et al., 2006). Axonal mitochondria display distinct motility patterns and undergo saltatory bidirectional movements where they stop and start moving, frequently changing direction. Whereas approximately one-third of axonal mitochondria is mobile in mature neurons, a large proportion remains stationary. Thus, efficient control of mitochondrial transport to and docking at particular sites of axons is likely essential for neuronal development and synaptic function. Mitochondrial balance between the motile and stationary phases is a possible target of regulating mitochondrial distribution. How are motile mitochondria recruited to the stationary pool in axons and at synapses? By generating a syntaphilin (snph) knock-out mouse, we recently discovered a novel role for SNPH as a "static anchor" for docking axonal mitochondria (Kang et al., 2008). SNPH specifically targets axonal mitochondria and mediates their docking in a stationary status by interacting with MTs. Such a mechanism enables neurons to maintain proper mitochondrial densities within axons and near synapses. However, the mecha- 
A

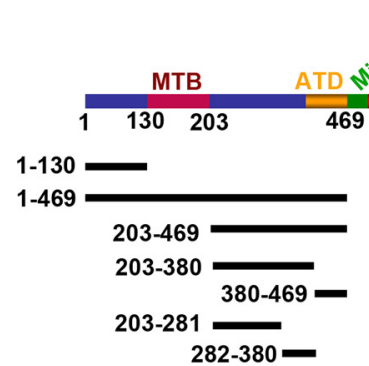

E

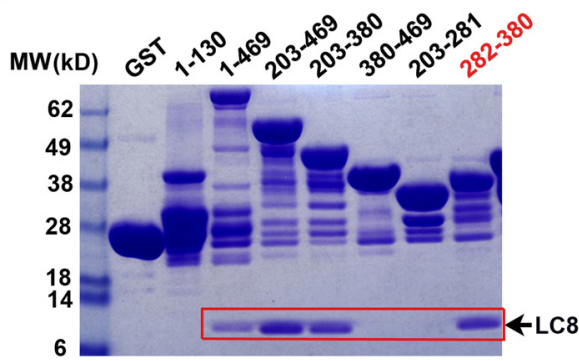

B

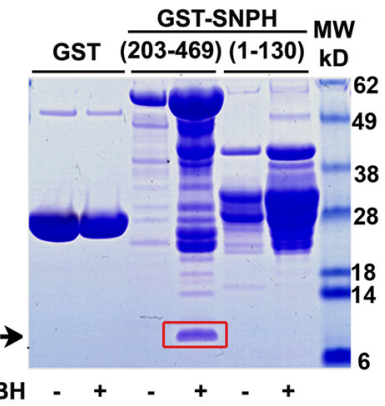

C
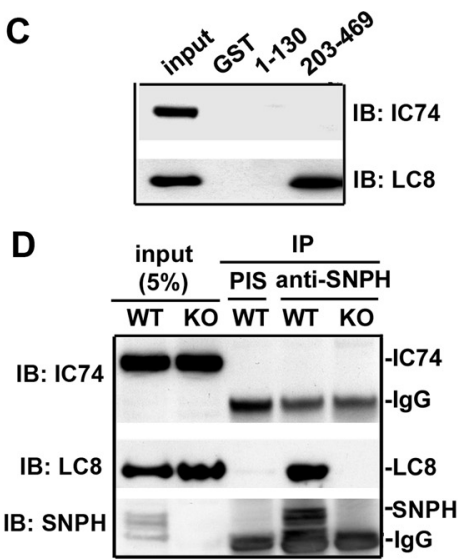

$\mathbf{F}$

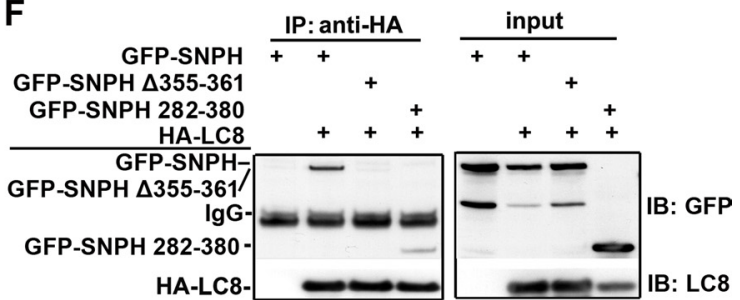

Figure 1. SNPH selectively interacts with LC8 via its seven-residue LC8-binding motif. $\boldsymbol{A}$, Schematic diagram of SNPH domain structures and truncated mutants used in GST pull-down study. MitoBD, Mitochondrial-binding domain. $\boldsymbol{B}$, Coomassie blue staining of GST pull-down from rat brain homogenates. GST-SNPH (203-469), but not (1-130) and GST control, pulled down a protein band at $10 \mathrm{kDa}$ (red box), which was then eluted for mass spectrometry and matches the sequences of rat LC8-1 and LC8-2. C, Immunoblot with anti-LC8 antibody confirmed the protein band as LC8. Dynein intermediate chain (IC74) was not detected in the same pull-down assay. D, Immunoprecipitation of SNPH with LC 8 from brain homogenates of snph wild-type mouse but not from snph $(-/-)$ mouse brain by an anti-SNPH antibody. IC74 was not detected from the same assays. Preimmune serum (PIS) was used as a control. $\boldsymbol{E}$, Pull-down assays revealed that the SNPH sequence from residues 282 to 380 is sufficient for stoichiometrical binding to LC8 (red box). $\boldsymbol{F}$, Immunoprecipitation of GFP-SNPH with HA-LC 8 from cotransfected COS7 cells further revealed a seven-residue sequence of SNPH (355-361) as the LC8-binding motif. Deleting this motif abolished its interaction with HA-LC8. MW, Molecular weight; HA, hemagglutinin; IP, immunoprecipitation; IB, immunoblot; BH, brain homogenates; WT, wild type; KO, knock-out.

nisms regulating SNPH-mediated mitochondrial anchoring at axons remain elusive.

In the current study, applying a proteomic approach of biochemical and cell biological assays combined with time-lapse imaging in live snph $(+/+)$ and $(-/-)$ neurons, we reveal that dynein light chain LC8 regulates axonal mitochondrial mobility through direct binding to the docking receptor SNPH, thus enhancing the SNPH-MT docking interaction. Our studies suggest an unexpected role for LC8 and provide new mechanistic insights into how SNPH and LC8 together immobilize mitochondria through a dynamic interaction between the docking receptor and axonal cytoskeleton.

\section{Materials and Methods}

DNA constructs. Various truncated and deleted SNPH mutants were generated by PCR and subcloned into pGEX-4T vector (GE Healthcare) for preparation of glutathione $S$-transferase (GST) fusion protein or into pET28 a vector for His-tagged fusion proteins (Novagen). The full-length encoding sequence of rat LC8 was cloned by PCR from rat brain marathon-ready DNA (Clontech) using primers (ATGTGCGACCGGAAGGCGGTG and TTAACCAGATTTGAACAGGAG), and then inserted into the vectors of pCMV-HA, pEGFP-C3, pIRES2-EGFP (Clontech) and pGEX-4T.

Recombinant protein preparation. GST-SNPH and GST-LC8 were prepared as described previously (Lao et al., 2000); the GST-tagged proteins were expressed in Escherichia coli BL21 (DE3). The BL21 cells were grown to an $\mathrm{OD}_{600}$ of 0.6 ; expressed protein was induced with isopropyl- $\beta$-D-thiogalactoside (IPTG) to a final concentration of $0.5 \mathrm{~mm}$ at $30^{\circ} \mathrm{C}$ for $4-6 \mathrm{~h}$. The cell pellet was resuspended in $20 \mathrm{~mm}$ phosphate buffer at $\mathrm{pH} 7.6$ containing $150 \mathrm{~mm} \mathrm{NaCl}, 0.5 \mathrm{~mm}$ DTT, and protease inhibitors. After sonication and centrifugation, the crude extract was purified using GST-Sepharose (GE Healthcare). The GST-tag was removed from the GST-fusion protein by Thrombin Kits (Novagen). Ex- pression of the His-tagged proteins His-SNPH (110-380) in bacteria and purification with Ni-NTA beads (Qiagen) were performed according to the manufacturer's instructions.

Isolation of SNPH-binding proteins using proteomic approach. The whole isolation procedure was performed at $4^{\circ} \mathrm{C}$. Rat brains were homogenized in Buffer A (25 mm Tris- $\mathrm{HCl}, \mathrm{pH} 7.5,50 \mathrm{~mm} \mathrm{NaCl}, 1 \mathrm{~mm}$ EDTA, 1 mм DTT, 0.5\% Triton X-100, 1 mm phenylmethylsulfonyl fluoride, $0.1 \mathrm{~mm}$ benzamidine, and $3 \mu \mathrm{g} / \mathrm{ml}$ each leupeptin and pepstatin). The crude homogenate was centrifuged at $15,000 \times g$ for $40 \mathrm{~min}$; the supernatant was carefully collected. GST beads coated with $10 \mu \mathrm{g}$ of GST, GST-SNPH (203-469), and GST-SNPH (1-130) were mixed with the lysates and incubated for $3 \mathrm{~h}$ with gentle rotation. The beads were then extensively washed with Buffer B (25 mM Tris- $\mathrm{HCl}, \mathrm{pH} 7.5,100 \mathrm{~mm}$ $\mathrm{NaCl}, 1$ mм DTT, 1 mm EDTA, 0.1\% Nonidet P-40, 1 mm phenylmethylsulfonyl fluoride, and $0.1 \mathrm{~mm}$ benzamidine). The beads were dissolved in SDS-PAGE sample buffer and boiled for $10 \mathrm{~min}$. Proteins were resolved by SDS-PAGE and visualized by Coomassie blue staining.

Coimmunoprecipitation. Mouse brains were homogenized in TBS with $1 \%$ Triton X-100/1\% sodium deoxycholate and protease inhibitors (1 mM phenylmethylsulfonyl fluoride, $0.1 \mathrm{~mm}$ benzamidine, and $3 \mu \mathrm{g} / \mathrm{ml}$ each leupeptin and pepstatin). The crude homogenate was centrifuged at $13,000 \times g$ for $30 \mathrm{~min}$ and the supernatant was collected. Brain homogenates were incubated with $3 \mu \mathrm{g}$ of rabbit anti-SNPH serum or rabbit preimmune serum in $0.5 \mathrm{ml}$ of TBS with $0.1 \%$ TX-100 and protease inhibitors, and incubated on a microtube rotator at $4^{\circ} \mathrm{C}$ for $1 \mathrm{~h}$. Protein A-Sepharose CL-4B resin (GE Healthcare) was added to each sample; incubation continued for an additional $3 \mathrm{~h}$, followed by three washes with TBS/0.1\% TX-100. The beads were dissolved in SDS-PAGE sample buffer and boiled for $10 \mathrm{~min}$. The proteins were resolved by SDS-PAGE and processed for Western blot analysis.

Mass spectrometry. Mass spectrometry was performed by the Protein Sequencing Facility at National Institute of Neurological Disorders and Stroke, National Institutes of Health. Protein bands excised from SDS- 

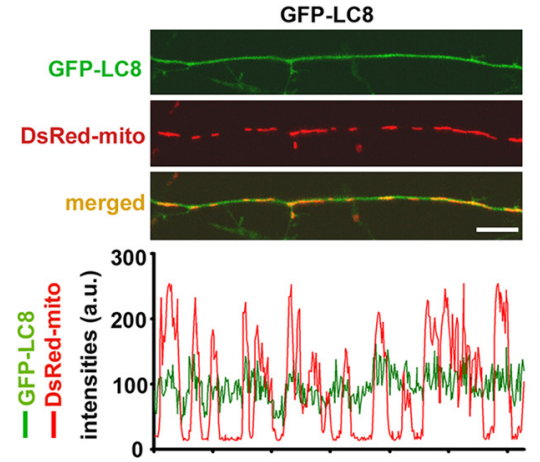

GFP-LC8 + SNPH
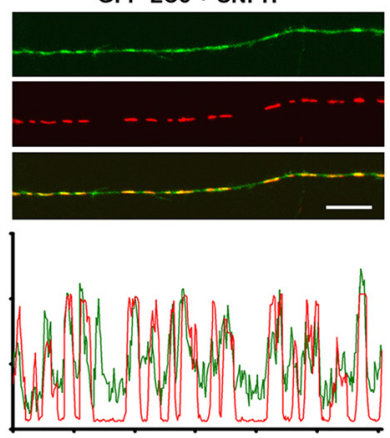

GFP-LC8 + SNPH $\triangle 355-361$

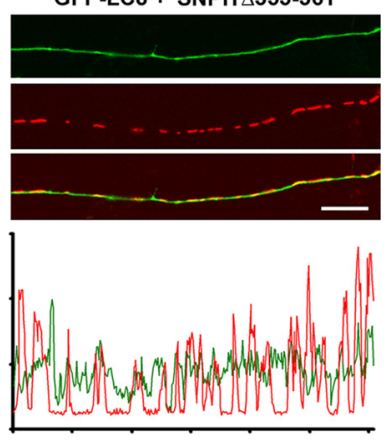

Figure 2. SNPH recruits LC8 to axonal mitochondria via its LC8-binding motif. Representative axonal images of hippocampal neurons cotransfected at DIV9 with GFP-LC8 and DsRed-mito (left), or combined with SNPH (middle) or SNPH $\Delta 355-361$ (right). Images were taken $4 \mathrm{~d}$ after transfection. The corresponding profiles (bottom panels) were plotted based on fluorescence intensities of GFP-LC8 and DsRed-mito and reflect the relative codistribution of LC8 (green) with mitochondria (red) along axonal processes. LC8, cytosolic protein, is diffused throughout the axonal cytoplasm. Coexpressing SNPH redistributes LC8 to mitochondria. Note that deleting the LC8-binding motif (SNPH $\Delta 355$-361) abolishes its role in recruiting GFP-LC8 to axonal mitochondria. a.u., Arbitrary units. Scale bars, $10 \mu \mathrm{m}$.

polyacrylamide gels were destained, reduced, alkylated, and then in-gel digested with trypsin. The resulting tryptic peptides were analyzed by liquid chromatography/mass spectroscopy/mass spectroscopy on a LTQ XL mass spectrometer (Thermo) equipped with an electrospray interface.

In vitro binding assay. A $10 \mu \mathrm{l}$ bed volume of glutathione-Sepharose resin was added to $20 \mu \mathrm{g}$ of GST-SNPH or its truncated and deleted mutants; the mixture was incubated on ice for $30 \mathrm{~min}$ before adding 10 $\mu \mathrm{g}$ of purified LC8. The mixture was incubated on ice for another $1-3 \mathrm{~h}$. The resin was then washed four times. Bound protein was eluted from the resin with $30 \mu \mathrm{l}$ of $2 \times$ SDS sample buffer and boiled for $10 \mathrm{~min}$. After centrifugation, the supernatant was subjected to SDS-PAGE followed by staining with Coomassie brilliant blue-G250.

Hippocampal neuron culture and transfection. Hippocampi were dissected from postnatal day 1 mouse or rat pups and cultured as previously described (Wilson et al., 2005). Briefly, after digestion with trypsin and triturating with fire-polished pipettes, hippocampal cells recovered by centrifugation were plated onto $12 \mathrm{~mm}$ coverslips; after $2 \mathrm{~h}, 2 \mathrm{ml}$ of plating medium was added to each $35 \mathrm{~mm}$ dish [for $100 \mathrm{ml}$ of plating medium: $89 \mathrm{ml}$ of Minimal Essential Medium (Invitrogen), $0.5 \mathrm{~g}$ of glucose, $0.5 \mathrm{~mm}$ glutamine, $2 \mathrm{~g}$ of $\mathrm{NaHCO}_{2}, 10 \mathrm{mg}$ of bovine transferrin (Calbiochem), $2.5 \mathrm{mg}$ of insulin, 10\% fetal calf serum]. From the second day in culture, half of the medium was replaced with feeding medium twice a week $[100 \mathrm{ml}$ of feeding medium: $97 \mathrm{ml}$ of minimal essential medium, $0.5 \mathrm{~g}$ of glucose, $0.5 \mathrm{~mm}$ glutamine, $2 \mathrm{~g}$ of $\mathrm{NaHCO}_{2}, 10 \mathrm{mg}$ of bovine transferring, $3 \mathrm{~mm}$ cytosine-p-arabinofuranoside (Sigma-Adrich) and 2\% B27 medium supplement (Invitrogen)]. DsRed-mito was used to label mitochondria (Millipore). Neurons were transfected at 9 days in vitro (DIV9) using the calcium phosphate method (Jiang and Chen, 2006), followed by time-lapse imaging 3-4 d after transfection.

Data acquisition and analysis for mitochondrial motility study. Timelapse imaging was performed using a Zeiss LSM 510 META confocal microscope (Zeiss) with a C-Apochromat $40 \times / 1.2 \mathrm{~W}$ Corr objective (Zeiss), and in the perfusion system $(0.4 \mathrm{ml}$ per minute) with modified Tyrode's solution (10 mM HEPES, $10 \mathrm{~mm}$ glucose, $3 \mathrm{~mm} \mathrm{KCl,} 145 \mathrm{~mm}$ $\mathrm{NaCl}, 1.2 \mathrm{~mm} \mathrm{CaCl}_{2}, 1.2 \mathrm{~mm} \mathrm{MgCl}_{2}$, and $1 \mu \mathrm{M}$ glycine, $\mathrm{pH}$ 7.4). Timelapse images were collected $\sim 512 \times 512$ pixel resolution ( 8 bit). A relatively long interval (10 s) for a total of 100 images was used to minimize laser-induced cellular damage. Kymographs were made with extra plugins for ImageJ (NIH) as previously described (Kang et al., 2008). A mitochondrion was considered stopped if it remained stationary for the entire recording period; a motile one was counted only if the displacement was at least $5 \mu \mathrm{m}$. The total number of mitochondria was defined as the number of mitochondria in each frame. Kymographs were used to trace the movement of mitochondria and count stationary ones. Counts for each axon were averaged from 11 frames for each time-lapse image to ensure accuracy and the relative percentage of stationary and motile events. Measurements not noted are presented as mean \pm SEM. Statistical analyses for unpaired $t$ tests were performed using software OriginPro (OriginLab).
Circular dichroism spectroscopy. Circular dichroism (CD) spectra were recorded on a Jasco J815 spectropolarimeter equipped with a Jasco PTC423S/15 temperature controller using a $1 \mathrm{~mm}$ cuvette and wavelength range between 190 and $250 \mathrm{~nm}$, as described previously (Greenfield, 2006). Samples for CD measurements were prepared in $20 \mathrm{~mm}$ phosphate buffer at pH 7.6 containing $150 \mathrm{~mm} \mathrm{NaCl}$ and $0.5 \mathrm{~mm}$ DTT at $20 \mu \mathrm{M}$ protein concentrations. For thermal denaturation experiments, protein samples were heated at a constant $1^{\circ} \mathrm{C} / \mathrm{min}$ rate and the $\mathrm{CD}$ signal was monitored at $222 \mathrm{~nm}$. Protein concentrations were determined spectrophotometrically and by densitometric analysis of Coomassie bluestained SDS-polyacrylamide gel compared with protein standards of known concentrations. The helical content, $\% \alpha$-helix, was estimated from the mean residue ellipticity, measured at $222 \mathrm{~nm}$ by using: $\% \alpha$-helix $=(-[\theta] 222 \mathrm{~nm}+3000) / 39,000$, where $[\theta]$ is a molar ellipticity (Morrow et al., 2000).

Spin-down assay of MT-associated proteins. GST-tagged SNPH 1-469 or GST-LC8 were purified by binding to glutathione-Sepharose beads (GE Healthcare) and eluted with $15 \mathrm{~mm}$ glutathione supplemented with $4 \mathrm{~mm}$ DTT and 2.5\% $\beta$-mercaptoethanol, and dialyzed in general tubulin buffer (80 mм PIPES pH 7, 5 mм $\mathrm{MgCl}_{2}, 1$ mм EGTA) supplemented with $4 \mathrm{~mm}$ DTT. The purified GST-tagged SNPH proteins were used for the MT spindown assay as described in the manual of the kit (Cytoskeleton). Briefly, $5 \mu \mathrm{g}$ of test protein were incubated in the absence or presence of $10 \mu \mathrm{g}$ of tubulin protein with $300 \mu \mathrm{M}$ taxol at room temperature for $30 \mathrm{~min}$. Each reaction was placed on top of the cushion buffer (80 mM PIPES pH 7, $5 \mathrm{~mm} \mathrm{MgCl}_{2}, 1$ mM EGTA, 50\% glycerol) and centrifuged at $100,000 \times g$ for $40 \mathrm{~min}$. The supernatant was removed from the top of each solution. The pellet in the bottom was resuspended in SDS sample buffer. Supernatants and pellets were analyzed by SDS-PAGE and visualized by Coomassie blue staining.

\section{Results}

\section{SNPH selectively interacts with LC8 via a seven-residue} LC8-binding motif

Our previous study identified SNPH domain sequences required for its association with mitochondria, axonal sorting, and MTbinding (Fig. 1A) (Kang et al., 2008). SNPH attaches to mitochondria through its $\mathrm{C}$ terminal domain (447-532), which is a moderately hydrophobic relatively short sequence with net positive charges flanking both sides, a signal structure conducive for mitochondrial outer-membrane-targeting (Rapaport, 2003). SNPH also targets to axons via its axon-targeting domain (ATD; residues 381-469). Expression of the SNPH mutant lacking ATD results in its distribution to all mitochondria, including those in the soma and dendrites. SNPH mediates mitochondrial docking via its microtubule-binding domain (MTB; residues 130-203), which has a high potential to form an $\alpha$-helical coiled-coil (Kang et al., 2008). 
To identify candidate proteins regulating SNPH-mediated axonal mitochondrial docking, we applied a proteomic approach using GST-tagged truncated SNPH mutants (Fig. 1A). The pulleddown candidate binding proteins from solubilized rat brain homogenates were visualized by Coomassie blue staining. SNPH truncated mutant (203-469), but not SNPH (1-130) and GST control, pulled down a protein band at $10 \mathrm{kDa}$ (Fig. $1 \mathrm{~B}$, red box), which was then eluted from the gel for mass spectrometry. The revealed sequences are consistent with rat dynein light chain (LC8-1 and 2) (GenBank accession number P63170 and Q78P75). To confirm the mass spectrometry results, we repeated the pull-down study followed by immunoblot analysis with anti-LC8 antibody, which specifically detected LC8 pulled down by GST-SNPH (203-469), but not by GST-SNPH (1-130) and GST (Fig. 1C).

Dynein is a multisubunit protein complex composed of heavy chains (HCs), intermediate chains (ICs), light intermediate chains (LICs), and light chains (LCs)

(Vallee et al., 2004). Whereas dynein HCs are polypeptides of $>500 \mathrm{kDa}$ responsible for ATPase and motor activities, other subunits of dynein complexes are presumed to promote assembly of the dynein complex and to be engaged in cargo attachment (Karki and Holzbaur, 1999; King et al., 2000; Barbar et al., 2001; Makokha et al., 2002). Three classes of LCs have been identified within cytoplasmic dynein: Tctex, LC8, and LC7. Dynein light chain 1 (LC1) and 2 (LC2) are highly conserved $10 \mathrm{kDa}$ light chains and belong to the LC8 family (Naisbitt et al., 2000; Wilson et al., 2001; Chuang et al., 2005). In addition to associating with the dynein motor complex, LC8 also interacts with a wide variety of proteins (Jaffrey and Snyder, 1996; Benashski et al., 1997; Puthalakath et al., 1999; Hays and Karess, 2000; Naisbitt et al., 2000; Raux et al., 2000; Schnorrer et al., 2000; Fuhrmann et al., 2002; Kaiser et al., 2003); a significant proportion of LC8 is widely distributed in cells and not associated with the dynein complex (King et al., 1996; Barbar, 2008), raising the possibility that LC8 functions independently of dynein-mediated transport.

Since LC8 was first described as a light-chain component of the dynein motor complex and LC8 binds to cytoplasmic dynein $\mathrm{HCs}$ through direct interaction with the ICs, we further tested whether LC8 bound to SNPH is in the same complex with dynein ICs. SNPH 203-469 pulled down LC8, but not IC74 (Fig. 1C). We further detected this complex in vivo using coimmunoprecipitation of brain homogenates from snph $(+/+)$ mouse with an antiSNPH antibody (Fig. 1D). However, the same antibody failed to pull down LC8 from snph (-/-) mouse brains, confirming the specificity of immunoprecipitation assays. Consistently, IC74 was undetectable under the same experimental conditions, further indicating that the LC8-SNPH interaction occurs independently of the dynein motor complex.

To determine the sequence required for this interaction, we conducted an in vitro binding assay with recombinant LC8 and GST-tagged truncated SNPH mutants. Coomassie blue staining of the pulled-down proteins identified that the SNPH sequence from residues 282 to 380 is sufficient for stoichimetrical binding to LC8 (Fig. $1 E$, red box). LC8 was reported to bind a wide range of proteins containing consensus peptide motifs, either "KXTQTX" or "XGIQVD," or a similar motif with conservative modifications. These motifs bind into two deep and opposing grooves formed by the stable LC8 homodimer (Lajoix et al., 2004). To further define the LC8-binding site, we searched for the SNPH sequence between residues 282 and 380 . Notably, SNPH has a close consensus LC8-binding motif "ERAIQTD” (residues 355-361) located between MTB and ATD domains. This motif is conserved in mammalian SNPH, including human, mouse, and rat. Deleting this seven-residue motif (GFP-SNPH $\Delta 355-361$ ) completely abolished coimmunoprecipitation of SNPH with LC8 from the cotransfected-COS cell lysates (Fig. $1 F$ ), indicating that SNPH selectively interacts with LC8 via its LC8-binding motif (ERAIQTD).

\section{SNPH recruits LC8 to axonal mitochondria via its LC8-binding motif}

As a soluble protein, LC8 is widely distributed throughout neuronal cytoplasm. To investigate whether SNPH recruits LC8 to axonal mitochondria via its LC8-binding motif, we cotransfected hippocampal neurons at DIV9 with DsRed-mito, green fluorescent protein (GFP)-LC8, and SNPH or SNPH $\Delta 355-361$ mutant, followed by imaging $4 \mathrm{~d}$ after transfection. Our previous study demonstrated that endogenous SNPH is relatively low; only $67 \%$ of axonal mitochondria under physiological conditions are labeled by SNPH (Kang et al., 2008). Thus, endogenous SNPH is not sufficient enough to recruit most over-expressed GFP-LC8 from the axonal cytoplasm to axonal mitochondria. Instead, we elevated SNPH expression to maximize redistribution of LC8, thus allowing us to detect the redistribution under confocal microscopy. GFP-LC8 showed diffuse cytoplasmic distribution along axons in the absence of exogenous expression of SNPH (Fig. 2). However, when SNPH was coexpressed, GFP-LC8 was efficiently recruited into the axonal mitochondria where SNPH is localized. Deleting the LC8-binding motif (SNPH $\Delta 355-361$ ) abolishes its recruiting capacity for GFP-LC8 to axonal mitochondria. The axonal imaging analysis supports our notion that SNPH recruits LC8 to axonal mitochondria via its LC8-binding 
A

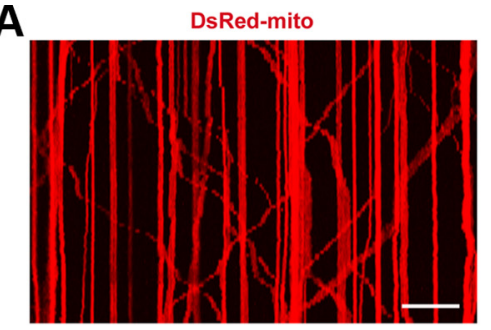

B

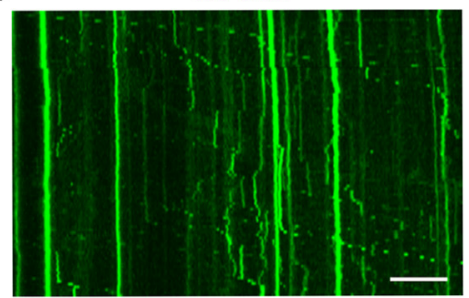

C

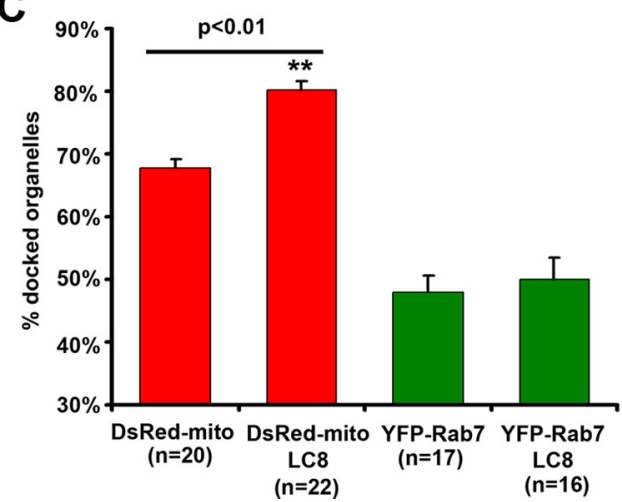

Figure 4. Elevated expression of $L C 8$ selectively inhibits the mobility of axonal mitochondria. $A$, Representative kymographs showing axonal mitochondrial mobility. Hippocampal neurons were transfected at DIV9 with DsRed-Mito (left) or cotransfected with DsRed-Mito and pIRES2-EGFP-LC8 (right). B, Representative kymographs showing late endosomal mobility in axons of hippocampal neurons transfected at DIV9 with YFP-Rab7 (left) or cotransfected with YFP-Rab7 and HA-LC8 (right). Axonal mitochondrial and late endosomal motility was observed by time-lapse imaging in live neurons $3 \mathrm{~d}$ after transfection. Scale bars, $20 \mu \mathrm{m}$. C, Relative docked axonal mitochondria (red) and late endosomes (green). Error bars: SEM; " $n$ " indicates the number of axons imaged. HA, Hemagglutinin. ${ }^{* *} p<0.01, t$ test.

A

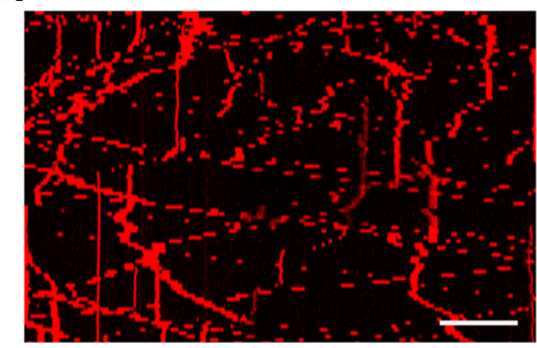

DsRed-mito pIRES2-EGFP-LC8
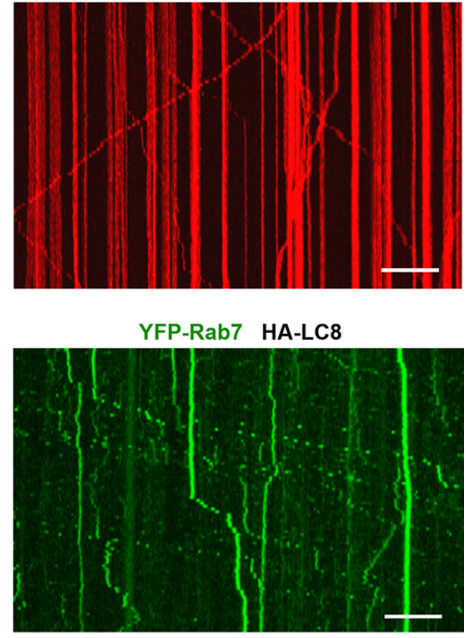

B DsRed-mito pIRES2-EGFP-LC8

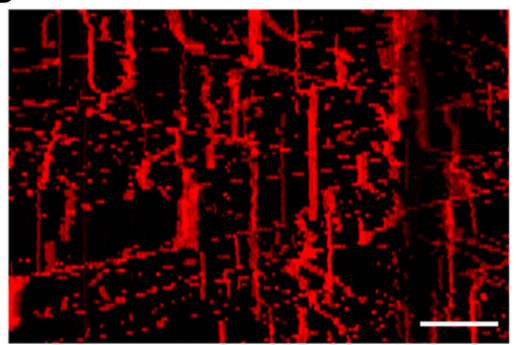

Figure 5. Role of LC8 in inhibiting mitochondrial mobility depends on SNPH. $A, B$, Representative kymographs showing axonal mitochondrial mobility in snph $(-/-)$ hippocampal neurons transfected at DIV9 with DsRed-Mito $(\boldsymbol{A})$ or cotransfected with DsRed-Mito and pIRES2-EGFP-LC8 (B). Neurons were time-lapse imaged $3 \mathrm{~d}$ posttransfection. Scale bars, $20 \mu \mathrm{m}$.

motif, which is consistent with our biochemical findings (Fig. $1 F$ ) that the seven-residue sequence (ERAIQTD) of SNPH is required for the SNPH-LC8 interaction and thus colocalization at axonal mitochondria.

Deleting the LC8-binding motif reduces the efficiency of exogenous SNPH in docking axonal mitochondria

To characterize the role of LC8-binding motif in mitochondrial docking efficiency, we conducted live cell time-lapse imaging us- ing confocal microscopy. Cultured hippocampal neurons were cotransfected with DsRed-mito and GFP-SNPH or GFP-SNPH $\Delta 355-361$ mutant at DIV9; axonal motile and stationary mitochondria were identified using kymographs $3 \mathrm{~d}$ after transfection, as previously described (Kang et al., 2008). Almost all axonal mitochondria labeled by GFP-SNPH remained stationary throughout our recording time (16 $\mathrm{min}$ ) (the percentage of docked mitochondria: $99.1 \pm 0.4 \%$, mean \pm SEM, 11 axons); only a few mitochondria not labeled by GFP-SNPH migrate along the axonal process (Fig. $3 A$, yellow arrow). In contrast, GFP-SNPH $\Delta 355-361$, although still targeted to axonal mitochondria, showed relatively lower docking efficiency. The percentage of docked axonal mitochondria labeled by GFP-SNPH $\Delta 355$ 361 is $93.1 \pm 1.0 \%$ (mean \pm SEM, $n=13$ axons, $p<0.01, t$ test) (Fig. $3 B$, white arrows). These indicate that deleting the LC8-binding motif impairs the docking efficiency of SNPH. The modest but significant effect observed suggests LC8 as a regulatory rather than essential protein in the SNPH-mediated docking mechanism. Alternatively, this modest effect may reflect the fact that the SNPH mutant only affects a small population (33\%) of axonal mitochondria not labeled by endogenous SNPH (Kang et al., 2008). Expressing SNPH mutant may have no effect on the docking efficiency of those mitochondria (67\%) associated with endogenous SNPH.

Elevated expression of LC8 selectively increases docking of axonal mitochondria through the

\section{SNPH-dependent mechanism}

To further determine the physiological relevance of LC8 in the mobility of axonal mitochondria in the presence and absence of endogenous SNPH, we transfected untagged LC8 (pIRES2-EGFP-LC8) in snph $(+/+)$ and $(-/-)$ hippocampal neurons at DIV9 followed by time-lapse imaging in live neurons $3 \mathrm{~d}$ after transfection. In wild-type cell expressing DsRed-mito alone, $67.8 \pm 1.3 \%$ (20 axons) of axonal mitochondria were stationary (Fig. $4 A$, $C$; supplemental Video $\mathrm{S} 1$, available at www.jneurosci.org as supplemental material). In contrast, LC8 over-expression resulted in significantly increased axonal mitochondria in docking status $(80.2 \pm 1.5 \%, 22$ axons, $p<0.01, t$ test) (Fig. 4A, C; supplemental Video S2, available at www. jneurosci.org as supplemental material). The density of axonal mitochondria was slightly increased when LC8 expression was elevated $(2.58 \pm 0.10$ per $10 \mu \mathrm{m}$ axon in length) relative to control ( $2.27 \pm 0.07, p<0.05, t$ test $)$, without affecting the ratio of retrograde to anterograde transport events of axonal 
mitochondria (control: $1.80 \pm 0.17$; LC8: $1.51 \pm 0.20, p>0.05)$.

SNPH acts as a docking receptor for axonal mitochondria. Deleting the mouse suph gene resulted in a robust increase in axonal mitochondrial motility; $18.4 \pm$ $3.1 \%$ (mean \pm SEM, $n=12$ axons) of axonal mitochondria remained in stationary status during the 16 min time-lapse recording (Fig. 5A; supplemental Video S3, available at www.jneurosci.org as supplemental material). However, in snph (-/-) neurons, overexpressing LC8 showed little effect on the mobility of axonal mitochondria, $22.1 \pm 3.8 \%(n=13$ axons, $p>0.05$ ) of axonal mitochondria remained stationary (Fig. 5B; supplemental Video S4, available at www.jneurosci. org as supplemental material). Thus, LC8 over-expression did not change axonal mitochondria motility in snph null neurons, suggesting that LC8-mediated inhibition of axonal mitochondrial mobility depends on SNPH expression. These results support a hypothesis that structural LC8-SNPH coupling is required to regulate mitochondrial docking in axons.

Given the fact that SNPH specifically targets to axonal mitochondria, not to other membranous organelles (Kang et al., 2008), it seems unlikely that LC8$\mathrm{SNPH}$ interaction has any impact on transport of other organelles. Considering LC8 as a dynein motor component, we performed two experiments to further address whether overexpressed LC8 plays a role in other organelle transport by affecting the motor proteins. First, we analyzed relative mobility of late endosomes [labeled by yellow fluorescent protein (YFP)-Rab7] along axonal processes of transfected neurons. Our study demonstrates that expressing LC8 in hippocampal neurons has no significant effect on the mobility of late endosomes (Fig. $4 B, C$ ). The relative docked late endosomes are $47.9 \pm 2.7 \%$ for control and $49.9 \pm 3.5 \%$ for LC8 expression ( $p>0.05)$. Second, we showed that expressing SNPH selectively immobilizes axonal mitochondria but not late endosomes under the same time-lapse images of transfected axons (supplemental Video S5, available at www.jneurosci.org as supplemental material), further supporting our notion that LC8SNPH interaction specifically regulates axonal mitochondrial docking.

\section{LC8 enhances SNPH-mediated docking via stabilizing the MT-binding domain of SNPH}

Although LC8 is an integral subunit of the dynein motor complex, recent reports suggest that LC8 may also play roles separate from the dynein motor. LC8 is widely distributed in cytoplasm and interacts with a variety of proteins; a significant proportion of LC8 is not associated with the dynein complex (Barbar, 2008). Our current results further support this notion. LC8 specifically binds to SNPH through the seven-residue LC8-binding motif, and such binding contributes to the regulation of axonal mito- chondrial mobility. To provide mechanistic insights into how LC8 modulates docking efficiency, we performed four lines of experiments.

First, we used COS7 cells instead of hippocampal neurons since axons are too thin to view two-dimensional arrays of MTs and to evaluate the relative distribution of LC8 in the cytoplasm versus targeting to MTs using confocal microscopy. Our previous study showed that GFP-SNPH (1-469), a mutant deleting the C terminal mitochondria-targeting domain, colocalized predominantly with microtubules in transfected COS7 cells. The pharmacological disruption of microtubules with Nocadazol or deletion of the MTB resulted in its diffusion throughout the cytoplasm (Kang et al., 2008). To determine whether LC8 and SNPH colocalize with MTs, we transfected COS7 cells with GFP-LC8 or GFP-SNPH (1-469), or cotransfected COS7 cells with GFP-LC8 and DsRed-SNPH (1-469) or its mutant lacking LC8-binding motif $(\Delta 355-361)$. Whereas GFP-LC8 is diffusely distributed in both cytoplasm and nuclei, GFP-SNPH (1-496) predominantly associates with MTs (Fig. 6A,B). However, when both proteins were coexpressed in COS7 cells, SNPH recruited the majority of GFP-LC8 to MTs, resulting in colocalization along MT fibers (Fig. 6C). Interestingly, targeting LC8 to MTs was abolished 
A
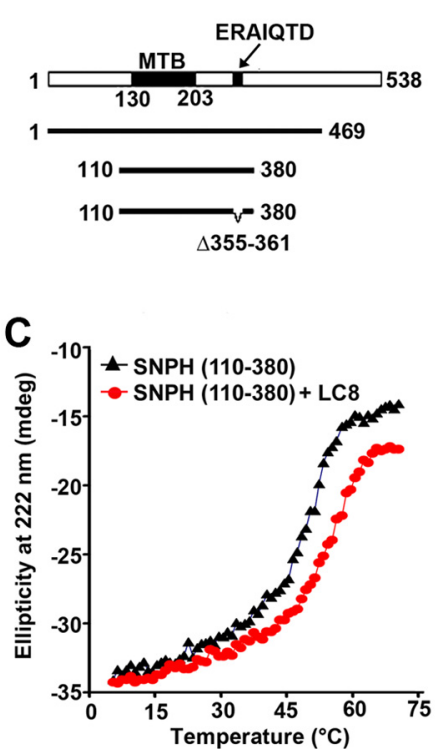
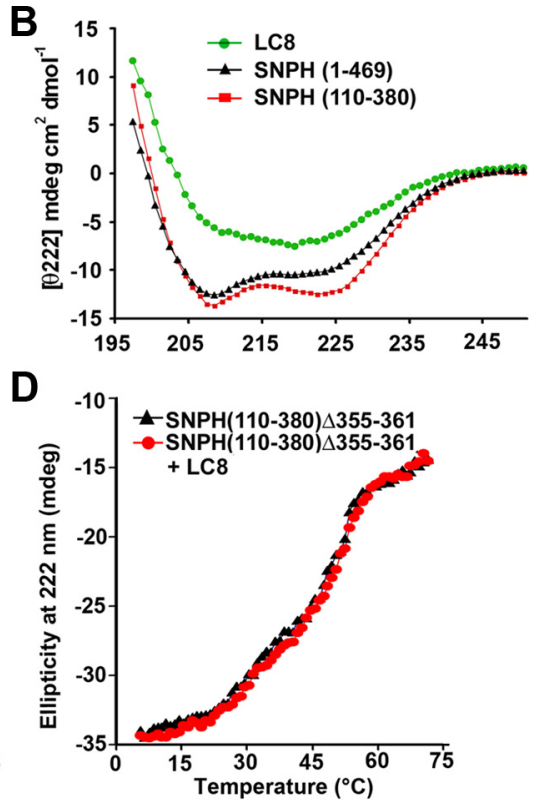

Figure 7. $\quad C D$ spectrum reveals the role of $L C 8$ in stabilizing the MT-binding domain of SNPH against thermal unfolding A, Schematic diagram of SNPH segments containing an $\alpha$-helical coiled-coil (130-203) MTB, and its mutant deleting the LC8-binding motif (355-361) used in far-UV CD measurements. $\boldsymbol{B}$, CD spectra of purified LC8, SNPH (1-469), and SNPH (110-380) at a concentration of $10 \mu \mathrm{m}$ in $20 \mathrm{~mm}$ phosphate buffer, $\mathrm{pH} 7.4$, containing $150 \mathrm{~mm} \mathrm{NaCl}$ and $0.5 \mathrm{~mm} \mathrm{DTT}$ at $25^{\circ} \mathrm{C}$ C, Thermal denaturation curves of SNPH (110-380) alone (black $\mathbf{\Delta})$ and mixture of SNPH (110-380) and LC8 after the subtraction of $L C 8$ spectrum (red $)$ ). $\boldsymbol{D}$, Thermal denaturation curves of $\mathrm{SNPH}(110-380)(\Delta 355-361)$ alone (black $\mathbf{\Delta})$ and a mixture of SNPH(110-380)( $\Delta 355-361)$ and $L C 8$ after the subtraction of LC spectrum (red $)$ ).

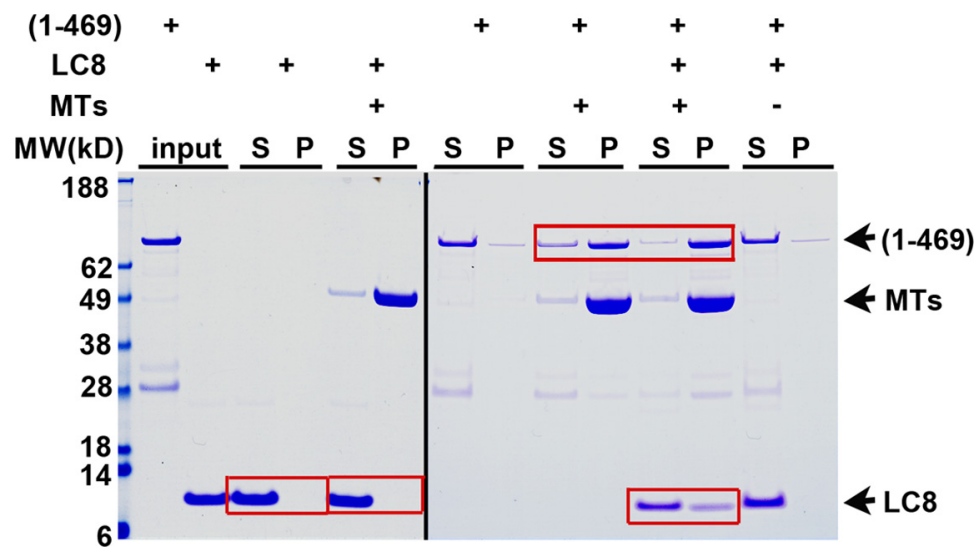

Figure 8. LC8 enhances cosedimentation of SNPH with MTs in spin-down assay. GST-SNPH (1-469) or the mixture of GST-SNPH (1-469) and LC 8 was incubated in the absence or presence of Taxol-stabilized MTs. Following centrifugation at 100,000 $\times g$ for 40 min, the supernatant $(S)$ and pellets $(P)$ were analyzed by SDS-PAGE and visualized by Coomassie blue staining. Note that LC8 remained in the supernatant in the absence of SNPH, whereas a significant part of SNPH (1-469) was spun down with MTs in the absence of LC8. Coincubating SNPH (1-469) and LC8 enhanced cosedimentation of both proteins with MTs (highlighted by red boxes). MW, Molecular weight.

when it was coexpressed with DsRed-SNPH $(1-469)(\Delta 355-361)$ (Fig. 6D), although the latter was still attached to MTs (data not shown). These imaging results provide evidence that LC8 associates with MTs via its binding to the LC8-binding motif of SNPH in intact cells.

Recent structural and thermodynamic characterization of the dynein motor complex suggests that LC8 facilitates the folding and increases the $\alpha$-helical content of the dynein ICs (Makokha et al., 2002; Nyarko et al., 2004; Williams et al., 2007; Barbar, 2008) and promotes the assembly of myosin motor coiled-coil domains (Wagner et al., 2006). Given that SNPH MT-binding domain contains a sequence (residues 130-203) with the potential to form an $\alpha$-helical coiled-coil, we asked whether LC8 binding could change the overall structure and thermodynamics of SNPH by applying far-ultraviolet (UV) CD measurements. The LC8 monomer has five $\beta$-strands and two $\alpha$-helices (Wilson et al., 2001). The CD spectrum of LC8 was similar to that previously reported for Drosophila LC8 (Barbar et al., 2001), representative of a typical $\alpha / \beta$ protein (Fig. 7B). However, the CD spectrum of SNPH 1-469 displayed single minima at $208 \mathrm{~nm}$ (Fig. $7 B$ ), and was not a typical $\alpha$-helical conformation. We also constructed a shorter segment of SNPH (110-380) including both the coiled-coil domain (130-203) and the LC8 binding motif (355-361) (Fig. 7A). Its CD spectrum showed double minima at 208 and $222 \mathrm{~nm}$ (Fig. $7 B$ ), typical for an $\alpha$-helical structure. At $25^{\circ} \mathrm{C}$, the calculated helical content was $\sim 40 \%$. The $\left[\theta_{222}\right] /\left[\theta_{208}\right]$ ratio for SNPH $(110-380)$ was 0.92 . A ratio close to 1 and higher indicates the existence of supercoiling (Wang et al., 2004).

To determine whether LC8 can stabilize the SNPH coiled-coil domain, we monitored changes in the $\mathrm{CD}$ signal of SNPH (110-380) during thermally induced unfolding in the presence and absence of LC8. Figure 7C shows thermal denaturation profiles at $222 \mathrm{~nm} \mathrm{SNPH}$ (110-380) (black $\boldsymbol{\Delta}$ ) and complex of SNPH (110-380) and LC8 after the subtraction of LC8 spectrum (red ). The progress curve at $222 \mathrm{~nm}$ for SNPH (110380) alone showed a loss of secondary structure as the temperature was increased. Strikingly, the complex of SNPH (110-380) and LC8 showed a broad, gradual thermal unfolding transition. The Tm value for SNPH (110-380) increased from $47^{\circ} \mathrm{C}$ in the absence of LC8 to $52^{\circ} \mathrm{C}$ in the presence of LC8. Because no significant thermal unfolding was observed for LC8 alone at temperatures up to $65^{\circ} \mathrm{C}$ (data not shown), we conclude that this increase in $\mathrm{Tm}$ is attributable to SNPH stabilization. To confirm LC8 binding attributes with the observed change in the thermal denaturation profiles, we used the SNPH (110-380) mutant deleting the LC8-binding motif (ERAIQTD) to prevent interaction of both proteins in the mixture (Fig. $7 \mathrm{~A}$ ). The thermal unfolding profile of SNPH $(110-380)(\Delta 355-361)$ is similar to that of SNPH (110-380) alone (Fig. 7D). No appreciable difference was observed between the thermal unfolding profiles of SNPH (110-380) $(\Delta 355-361)$ in the absence (black $\boldsymbol{\Delta}$ ) and presence (red ) of LC8 after the subtraction of LC8 spectrum. These observations indicate that the observed change in the thermal unfolding profile of SNPH (110-380) is attributable to direct LC8 binding but not to molecular crowding. CD analysis suggests 
that the SNPH MT-binding domain forms an unstable $\alpha$-helical structure, whereas LC8 serves a "stabilizer" of SNPH coiled-coil domain required for highaffinity interaction with MTs.

Next, we asked whether the observed change in the thermal dynamics of SNPH during LC8 binding affects the SNPH-MT docking interaction. We performed in vitro spin-down assays using Taxol-stabilized MTs to pellet MTs at a high centrifugation speed. Any protein that associates with MTs will be found in the pellet because of cosedimentation. A representative SDSPAGE analysis of the supernatant versus pellet fraction is shown in Figure 8. LC8 is not a microtubule-associated protein, since it remained in the supernatant after centrifugation. Whereas the majority of SNPH $(75.5 \pm 3.7 \%$, mean \pm SEM, $n=4$ ) was spun down with MTs in the pellet in the absence of LC8, LC8 further enhanced cosedimentation of SNPH with MTs $(90.7 \pm 3.1 \%, n=4, p<0.01)$. In addition, a significant portion $(30 \%)$ of LC8 was also found in the pellet with MTs in the presence of SNPH. Altogether, our cell biology and biochemical approaches combined with time-lapse imaging analysis reveal an unexpected role for LC8 in enhancing SNPH-mediated mitochondrial docking in axons via stabilizing its MT-binding domain.

\section{Discussion}

In the current study, we sought to identify proteins regulating mitochondrial docking in axons. By applying a proteomic approach and cell biological assays combined with time-lapse imaging in live neurons, we reveal that LC8 enhances the SNPH-mediated mitochondrial docking efficiency through modulating the dynamic interaction between the docking receptor and axonal cytoskeleton. Four lines of evidence support this view. First, SNPH selectively interacted with LC8 via its seven-residue LC8-binding motif (ERAIQTD). The SNPH-LC8 complex was detected in vivo by coimmunoprecipitation of brain homogenates from $s n p h$ wild-type but not snph $(-/-)$ mice; the interaction occurred independently of the dynein motor complex. Second, SNPH recruited LC8 to axonal mitochondria via its LC8-binding motif. Deleting this motif reduced the efficiency of SNPH in docking axonal mitochondria. Furthermore, elevated LC8 expression inhibited the mobility of axonal mitochondria through endogenous SNPH. LC8 over-expression did not change axonal mitochondria motility in snph null neurons, suggesting that the LC8 reduces axonal mitochondrial mobility in a manner depending on its physical coupling with SNPH. Finally, CD spectrum analysis revealed that LC8 enhances SNPH-mediated docking by stabilizing its MT-binding domain. Our studies suggest an unexpected role for LC8 and provide new mechanistic insights into how SNPH and LC8 together immobilize mitochondria through a dynamic interaction between the docking receptor and axonal cytoskeleton.

LC8 was first described as a light-chain component of the Chlamydomonas outer dynein arm and later a component of mammalian flagellar and cytoplasmic dyneins (King and PatelKing, 1995; Dick et al., 1996). In addition to its association with ICs at the cargo-binding tail region of the dynein motor complex (Lo et al., 2001), LC8 has been shown to bind to diverse targets including transcription factors $\mathrm{p}^{53}$-binding protein 1 (53BP1) (Lo et al., 2005) and Swallow (Schnorrer et al., 2000), signaling molecules Bim (Puthalakath et al., 1999), scaffolding proteins gephyrin (Fuhrmann et al., 2002), and guanylate kinase domainassociated protein (GKAP) (Naisbitt et al., 2000). One interpretation for these interactions is that LC8 links these cargo proteins to the dynein motor (Navarro-Lérida et al., 2004). However, the physiological relevance of these interactions on microtubulebased transport remains to be determined. Alternatively, LC8 has been shown to regulate protein function in a dynein-independent manner. For instance, LC8 binding inhibits TNF $\alpha$-induced nuclear factor $-\kappa \mathrm{B}$ activation by interacting with $\mathrm{I} \kappa \mathrm{B} \alpha$, thereby preventing its phosphorylation by $\mathrm{I} \kappa \mathrm{B}$ kinase (IKK) (Jung et al., 2008). LC8 can interact with transcription factor TRPS1 and suppress its transcriptional repression activity (Kaiser et al., 2003). Identification of LC8 as a structural regulator of SNPH-mediated mitochondrial docking machinery further highlights its role as a multivalent effector in broader intracellular events, in addition to its connection with dynein motor or microtubule-based transport.

We applied three alternative approaches to exclude any pleiotropic effect caused by exogenously expressed LC8 in neurons and COS cells. First, we used the dominant-negative SNPH mutant lacking seven-residue LC8 binding motif $(\Delta 355-361)$ to confirm our findings both in vitro (Fig. $1 F$ ) and in transfected cells (Figs. 2, 3, 6D, 7). Second, instead of using recombinant proteins, we confirmed the SNPH-LC8 interaction in brain homogenates, which demonstrates that SNPH was coimmunoprecipitated with LC8 from brain homogenates of snph wild-type mouse but not from snph $(-/-)$ mouse brain by an anti-SNPH antibody (Fig. $1 D)$. Finally, we further verified that transient LC8 expression reduces mitochondrial mobility in snph $(+/+)$ but not $(-/-)$ neurons (Figs. 4, 5), suggesting that the observed effect of LC8 specifically depends on the SNPH-mediated docking mechanism.

Two consensus LC8-binding sequences (GIQVD and KSTQT) were identified (Rodríguez-Crespo et al., 2001), where Gln (Q) is the most critical residue for LC8 interaction. The residues at positions -1 and +1 (flanking Q) consist of hydrophobic residues (Thr, Val) that can only be replaced by hydrophobic or aromatic amino acids. Position -4 (Asp or Ser) may also be important for binding (Lajoix et al., 2004). The LC8-binding motif identified in SNPH, ERAIQTD, is similar to one of the sequence structures (GIQVD). Structural investigations showed that each characterized LC8 target binds to the same site as the dynein IC (Liang et al., 1999). Although the LC8-binding (TQT) peptide readily binds free LC8, it cannot bind to dynein-associated LC8 (Bergen and Pun, 2007). Consistently, our pull-down and coimmunoprecipitation assays demonstrated that SNPH interacts with LC8 in 
vitro or forms a complex in brain homogenates independently of dynein IC.

Recent structural and thermodynamic characterization of the dynein motor complex suggests that LC8 facilitates the folding and increases the $\alpha$-helical content of the dynein ICs (Makokha et al., 2002; Nyarko et al., 2004; Williams et al., 2007; Barbar, 2008). LC8 presents as dimmer in 1:1 stoichiometry with two copies of ICs. The dynein IC is intrinsically unstructured and highly disordered protein. During binding to LC8, it undergoes a significant conformational change to a more ordered structure. CD and fluorescence spectroscopy analysis demonstrated an $8 \%$ increase in the helical content and a gain of compact structure (Makokha et al., 2002; Nyarko et al., 2004). In addition, LC8 promoted assembly of the coiled-coil domain of swallow protein (Wang et al., 2004) and stabilized a portion of myosin's coiled-coil domain (Wagner et al., 2006). Our study showed that LC8 binds to SNPH through the conservative LC8-binding motif, stabilizes the SNPH coiled-coil structure within the MT-binding domain, thus increasing the binding affinity of SNPH with microtubules. All these findings favor a model in which LC8 serves as the "stabilizer" of a helix coiled-coil structure of mitochondrial docking/ anchoring receptor SNPH. Such a physical coupling between LC8 and SNPH may control mitochondrial mobility and density in axons and at synapses (Fig. 9). Our model is consistent with the accumulated evidence that as a hub protein LC8 is essential in diverse protein networks by stabilizing the helix structure of partially disordered proteins (Barbar, 2008).

The coordination of mitochondrial mobility/docking with axonal physiology is crucial for neuronal and synaptic function. Axonal and synaptic structure and function are highly plastic and undergo spontaneous and activity-dependent remodeling, thereby changing the demand for mitochondria. Motile mitochondria are recruited to the stationary pool near synapses in response to elevated cytosolic $\mathrm{Ca}^{2+}$ and synaptic activity (Rintoul et al., 2003; Li et al., 2004; Yi et al., 2004). Identification of mitochondrial motor-adaptor transport complexes KIF5-Milton-Miro (Stowers et al., 2002; Glater et al., 2006) and KIF5-syntabulin (Cai et al., 2005) provide molecular targets for such regulation. Recent studies from three groups independently provide compelling evidence that Miro serves as a calcium sensor that controls mitochondrial movement (Saotome et al., 2008; Macaskill et al., 2009; Wang and Schwarz, 2009). Our previous study elucidates $\mathrm{SNPH}$ as an anchoring receptor for docking mitochondria in axons via interacting with microtubule-based cytoskeleton (Kang et al., 2008). Such a mechanism enables neurons to maintain proper densities of stationary mitochondria within axons and in the proximity of synapses. Docked stationary mitochondria ideally serve as local energy stations by providing ATP to sustain the high activity of $\mathrm{Na}^{+}-\mathrm{K}^{+}$ATPase and fast spike propagation and maintain $\mathrm{Ca}^{2+}$ homeostasis to support synaptic transmission. Loss of mitochondria at axon terminals results in impaired synaptic transmission (Stowers et al., 2002; Guo et al., 2005; Verstreken et al., 2005). Defective transport of axonal mitochondria is implicated in human neurological disorders and neurodegenerative diseases (Hirokawa and Takemura, 2004; Chan, 2006; Stokin and Goldstein, 2006). It is expected that defective docking/ anchoring machinery could affect normal neuronal functions, particularly for cells with long axonal processes such as motor neurons.

In summary, our current study provides mechanistic insights into coordinated regulation of mitochondrial anchoring in axons by LC8 and SNPH. Identification of LC8 as a stabilizer of SNPH-MT docking interaction provides a molecular target for regulating axonal mitochondrial motility. Future studies using genetic mice models will provide molecular and cellular details on how LC8-SNPH regulates mitochondrial balance between motile and stationary phases in response to the diverse and dynamic physiological states of axons and synapses.

\section{References}

Barbar E (2008) Dynein light chain LC8 is a dimerization hub essential in diverse protein networks. Biochemistry 47:503-508.

Barbar E, Kleinman B, Imhoff D, Li M, Hays TS, Hare M (2001) Dimerization and folding of LC8, a highly conserved light chain of cytoplasmic dynein. Biochemistry 40:1596-1605.

Benashski SE, Harrison A, Patel-King RS, King SM (1997) Dimerization of the highly conserved light chain shared by dynein and myosin V. J Biol Chem 272:20929-20935.

Bergen JM, Pun SH (2007) Evaluation of an LC8-binding peptide for the attachment of artificial cargo to dynein. Mol Pharm 4:119-128.

Billups B, Forsythe ID (2002) Presynaptic mitochondrial calcium sequestration influences transmission at mammalian central synapses. J Neurosci 22:5840-5847.

Cai Q, Gerwin C, Sheng ZH (2005) Syntabulin-mediated anterograde transport of mitochondria along neuronal processes. J Cell Biol 170:959-969.

Chan DC (2006) Mitochondria: dynamic organelles in disease, aging, and development. Cell 125:1241-1252.

Chuang JZ, Yeh TY, Bollati F, Conde C, Canavosio F, Caceres A, Sung CH (2005) The dynein light chain Tctex-1 has a dynein-independent role in actin remodeling during neurite outgrowth. Dev Cell 9:75-86.

Dick T, Ray K, Salz HK, Chia W (1996) Cytoplasmic dynein (ddlc1) mutations cause morphogenetic defects and apoptotic cell death in Drosophila melanogaster. Mol Cell Biol 16:1966-1977.

Fuhrmann JC, Kins S, Rostaing P, El Far O, Kirsch J, Sheng M, Triller A, Betz H, Kneussel M (2002) Gephyrin interacts with Dynein light chains 1 and 2, components of motor protein complexes. J Neurosci 22:5393-5402.

Glater EE, Megeath LJ, Stowers RS, Schwarz TL (2006) Axonal transport of mitochondria requires milton to recruit kinesin heavy chain and is light chain independent. J Cell Biol 173:545-557.

Górska-Andrzejak J, Stowers RS, Borycz J, Kostyleva R, Schwarz TL, Meinertzhagen IA (2003) Mitochondria are redistributed in Drosophila photoreceptors lacking milton, a kinesin-associated protein. J Comp Neurol 463:372-388

Greenfield NJ (2006) Using circular dichroism spectra to estimate protein secondary structure. Nat Protoc 1:2876-2890.

Guo X, Macleod GT, Wellington A, Hu F, Panchumarthi S, Schoenfield M, Marin L, Charlton MP, Atwood HL, Zinsmaier KE (2005) The GTPase dMiro is required for axonal transport of mitochondria to Drosophila synapses. Neuron 47:379-393.

Hays T, Karess R (2000) Swallowing dynein: a missing link in RNA localization? Nat Cell Biol 2:E60-E62.

Hirokawa N, Takemura R (2004) Molecular motors in neuronal development, intracellular transport and diseases. Curr Opin Neurobiol 14:564-573.

Hollenbeck PJ (1996) The pattern and mechanism of mitochondrial transport in axons. Front Biosci 1:d91-d102.

Hollenbeck PJ, Saxton WM (2005) The axonal transport of mitochondria. J Cell Sci 118:5411-5419.

Jaffrey SR, Snyder SH (1996) PIN: an associated protein inhibitor of neuronal nitric oxide synthase. Science 274:774-777.

Jiang M, Chen G (2006) High Ca2+-phosphate transfection efficiency in low-density neuronal cultures. Nat Protoc 1:695-700.

Jung Y, Kim H, Min SH, Rhee SG, Jeong W (2008) Dynein light chain LC8 negatively regulates NF-kappaB through the redox-dependent interaction with IkappaBalpha. J Biol Chem 283:23863-23871.

Kaiser FJ, Tavassoli K, Van den Bemd GJ, Chang GT, Horsthemke B, Möröy T, Lüdecke HJ (2003) Nuclear interaction of the dynein light chain LC8a with the TRPS1 transcription factor suppresses the transcriptional repression activity of TRPS1. Hum Mol Genet 12:1349-1358.

Kang JS, Tian JH, Pan PY, Zald P, Li C, Deng C, Sheng ZH (2008) Docking of axonal mitochondria by syntaphilin controls their mobility and affects short-term facilitation. Cell 132:137-148.

Karki S, Holzbaur EL (1999) Cytoplasmic dynein and dynactin in cell division and intracellular transport. Curr Opin Cell Biol 11:45-53.

King JM, Hays TS, Nicklas RB (2000) Dynein is a transient kinetochore 
component whose binding is regulated by microtubule attachment, not tension. J Cell Biol 151:739-748.

King SM, Patel-King RS (1995) The M(r) $=8000$ and 11,000 outer arm dynein light chains from Chlamydomonas flagella have cytoplasmic homologues. J Biol Chem 270:11445-11452.

King SM, Barbarese E, Dillman JF 3rd, Patel-King RS, Carson JH, Pfister KK (1996) Brain cytoplasmic and flagellar outer arm dyneins share a highly conserved Mr 8,000 light chain. J Biol Chem 271:19358-19366.

Lajoix AD, Gross R, Aknin C, Dietz S, Granier C, Laune D (2004) Cellulose membrane supported peptide arrays for deciphering protein-protein interaction sites: the case of PIN, a protein with multiple natural partners. Mol Divers 8:281-290.

Lao G, Scheuss V, Gerwin CM, Su Q, Mochida S, Rettig J, Sheng ZH (2000) Syntaphilin: a syntaxin-1 clamp that controls SNARE assembly. Neuron 25:191-201.

Levy M, Faas GC, Saggau P, Craigen WJ, Sweatt JD (2003) Mitochondrial regulation of synaptic plasticity in the hippocampus. J Biol Chem 278:17727-17734.

Li Z, Okamoto K, Hayashi Y, Sheng M (2004) The importance of dendritic mitochondria in the morphogenesis and plasticity of spines and synapses. Cell 119:873-887.

Liang J, Jaffrey SR, Guo W, Snyder SH, Clardy J (1999) Structure of the PIN/LC8 dimer with a bound peptide. Nat Struct Biol 6:735-740.

Ligon LA, Steward O (2000) Movement of mitochondria in the axons and dendrites of cultured hippocampal neurons. J Comp Neurol 427:340-350.

Lo KW, Naisbitt S, Fan JS, Sheng M, Zhang M (2001) The 8-kDa dynein light chain binds to its targets via a conserved (K/R)XTQT motif. J Biol Chem 276:14059-14066.

Lo KW, Kan HM, Chan LN, Xu WG, Wang KP, Wu Z, Sheng M, Zhang M (2005) The 8-kDa dynein light chain binds to p53-binding protein 1 and mediates DNA damage-induced p53 nuclear accumulation. J Biol Chem 280:8172-8179.

Macaskill AF, Rinholm JE, Twelvetrees AE, Arancibia-Carcamo IL, Muir J, Fransson A, Aspenstrom P, Attwell D, Kittler JT (2009) Mirol is a calcium sensor for glutamate receptor-dependent localization of mitochondria at synapses. Neuron 61:541-555.

Makokha M, Hare M, Li M, Hays T, Barbar E (2002) Interactions of cytoplasmic dynein light chains Tctex-1 and LC8 with the intermediate chain IC74. Biochemistry 41:4302-4311.

Martin M, Iyadurai SJ, Gassman A, Gindhart JG Jr, Hays TS, Saxton WM (1999) Cytoplasmic dynein, the dynactin complex, and kinesin are interdependent and essential for fast axonal transport. Mol Biol Cell 10:3717-3728.

Morrow JA, Segall ML, Lund-Katz S, Phillips MC, Knapp M, Rupp B, Weisgraber KH (2000) Differences in stability among the human apolipoprotein $\mathrm{E}$ isoforms determined by the amino-terminal domain. Biochemistry 39:11657-11666.

Naisbitt S, Valtschanoff J, Allison DW, Sala C, Kim E, Craig AM, Weinberg RJ, Sheng M (2000) Interaction of the postsynaptic density-95/guanylate kinase domain-associated protein complex with a light chain of myosin- $\mathrm{V}$ and dynein. J Neurosci 20:4524-4534.

Navarro-Lérida I, Martínez Moreno M, Roncal F, Gavilanes F, Albar JP, Rodríguez-Crespo I (2004) Proteomic identification of brain proteins that interact with dynein light chain LC8. Proteomics 4:339-346.

Nyarko A, Hare M, Hays TS, Barbar E (2004) The intermediate chain of cytoplasmic dynein is partially disordered and gains structure upon binding to light-chain LC8. Biochemistry 43:15595-15603.

Pilling AD, Horiuchi D, Lively CM, Saxton WM (2006) Kinesin-1 and Dynein are the primary motors for fast transport of mitochondria in Drosophila motor axons. Mol Biol Cell 17:2057-2068.

Puthalakath H, Huang DC, O'Reilly LA, King SM, Strasser A (1999) The proapoptotic activity of the Bcl-2 family member Bim is regulated by interaction with the dynein motor complex. Mol Cell 3:287-296.
Rapaport D (2003) Finding the right organelle. Targeting signals in mitochondrial outer-membrane proteins. EMBO Rep 4:948-952.

Raux H, Flamand A, Blondel D (2000) Interaction of the rabies virus P protein with the LC8 dynein light chain. J Virol 74:10212-10216.

Rintoul GL, Filiano AJ, Brocard JB, Kress GJ, Reynolds IJ (2003) Glutamate decreases mitochondrial size and movement in primary forebrain neurons. J Neurosci 23:7881-7888.

Rodríguez-Crespo I, Yélamos B, Roncal F, Albar JP, Ortiz de Montellano PR, Gavilanes F (2001) Identification of novel cellular proteins that bind to the LC8 dynein light chain using a pepscan technique. FEBS Lett 503:135-141.

Saotome M, Safiulina D, Szabadkai G, Das S, Fransson A, Aspenstrom P, Rizzuto R, Hajnóczky G (2008) Bidirectional Ca2+-dependent control of mitochondrial dynamics by the Miro GTPase. Proc Natl Acad Sci U S A 105:20728-20733.

Schnorrer F, Bohmann K, Nüsslein-Volhard C (2000) The molecular motor dynein is involved in targeting swallow and bicoid RNA to the anterior pole of Drosophila oocytes. Nat Cell Biol 2:185-190.

Stokin GB, Goldstein LS (2006) Axonal transport and Alzheimer's disease. Annu Rev Biochem 75:607-627.

Stowers RS, Megeath LJ, Górska-Andrzejak J, Meinertzhagen IA, Schwarz TL (2002) Axonal transport of mitochondria to synapses depends on milton, a novel Drosophila protein. Neuron 36:1063-1077.

Tanaka Y, Kanai Y, Okada Y, Nonaka S, Takeda S, Harada A, Hirokawa N (1998) Targeted disruption of mouse conventional kinesin heavy chain, kif5B, results in abnormal perinuclear clustering of mitochondria. Cell 93:1147-1158.

Tang Y, Zucker RS (1997) Mitochondrial involvement in post-tetanic potentiation of synaptic transmission. Neuron 18:483-491.

Vallee RB, Williams JC, Varma D, Barnhart LE (2004) Dynein: An ancient motor protein involved in multiple modes of transport. J Neurobiol 58:189-200.

Verstreken P, Ly CV, Venken KJ, Koh TW, Zhou Y, Bellen HJ (2005) Synaptic mitochondria are critical for mobilization of reserve pool vesicles at Drosophila neuromuscular junctions. Neuron 47:365-378.

Wagner W, Fodor E, Ginsburg A, Hammer JA 3rd (2006) The binding of DYNLL2 to myosin Va requires alternatively spliced exon $\mathrm{B}$ and stabilizes a portion of the myosin's coiled-coil domain. Biochemistry 45:11564-11577.

Wang L, Hare M, Hays TS, Barbar E (2004) Dynein light chain LC8 promotes assembly of the coiled-coil domain of swallow protein. Biochemistry 43:4611-4620.

Wang X, Schwarz TL (2009) The mechanism of Ca2+-dependent regulation of kinesin-mediated mitochondrial motility. Cell 136:163-174.

Werth JL, Thayer SA (1994) Mitochondria buffer physiological calcium loads in cultured rat dorsal root ganglion neurons. J Neurosci 14:348-356.

Williams JC, Roulhac PL, Roy AG, Vallee RB, Fitzgerald MC, Hendrickson WA (2007) Structural and thermodynamic characterization of a cytoplasmic dynein light chain-intermediate chain complex. Proc Natl Acad Sci U S A 104:10028-10033.

Wilson MJ, Salata MW, Susalka SJ, Pfister KK (2001) Light chains of mammalian cytoplasmic dynein: identification and characterization of a family of LC8 light chains. Cell Motil Cytoskeleton 49:229-240.

Wilson NR, Kang J, Hueske EV, Leung T, Varoqui H, Murnick JG, Erickson JD, Liu G (2005) Presynaptic regulation of quantal size by the vesicular glutamate transporter VGLUT1. J Neurosci 25:6221-6234.

Yang F, He XP, Russell J, Lu B (2003) Ca2+ influx-independent synaptic potentiation mediated by mitochondrial $\mathrm{Na}(+)-\mathrm{Ca} 2+$ exchanger and protein kinase C. J Cell Biol 163:511-523.

Yi M, Weaver D, Hajnóczky G (2004) Control of mitochondrial motility and distribution by the calcium signal: a homeostatic circuit. J Cell Biol $167: 661-672$. 